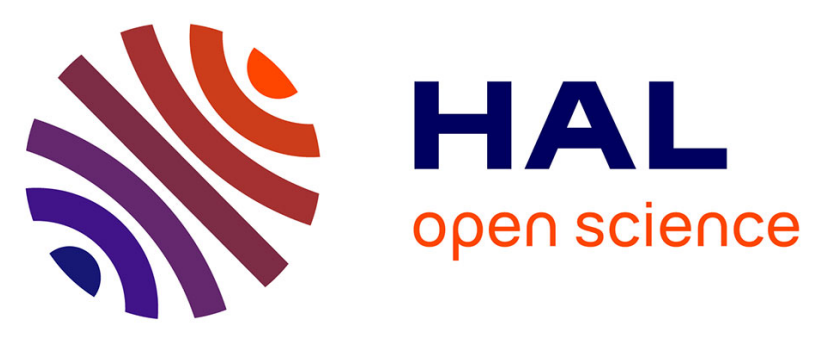

\title{
Prospective comparison of the Montreal Cognitive Assessment (MoCA) and the Mini Mental State Examination (MMSE) in geriatric oncology
}

Audrey Rambeau, Bérengère Beauplet, Heidi Laviec, Idlir Licaj, Alexandra Leconte, Claire Chatel, Priscille Le Bon, Julie Denhaerynck, Bénédicte Clarisse, Nicole Frenkiel, et al.

\section{To cite this version:}

Audrey Rambeau, Bérengère Beauplet, Heidi Laviec, Idlir Licaj, Alexandra Leconte, et al.. Prospective comparison of the Montreal Cognitive Assessment (MoCA) and the Mini Mental State Examination (MMSE) in geriatric oncology. Journal of Geriatric Oncology, 2019, 10 (2), pp.235-240. 10.1016/j.jgo.2018.08.003 . inserm-02100129

HAL Id: inserm-02100129

https://www.hal.inserm.fr/inserm-02100129

Submitted on 22 Oct 2021

HAL is a multi-disciplinary open access archive for the deposit and dissemination of scientific research documents, whether they are published or not. The documents may come from teaching and research institutions in France or abroad, or from public or private research centers.
L'archive ouverte pluridisciplinaire HAL, est destinée au dépôt et à la diffusion de documents scientifiques de niveau recherche, publiés ou non, émanant des établissements d'enseignement et de recherche français ou étrangers, des laboratoires publics ou privés.

\section{(ㄷ)(1) $\$$}

Distributed under a Creative Commons Attribution - NonCommerciall 4.0 International 
Version of Record: https://www.sciencedirect.com/science/article/pii/S187940681830095X

Manuscript_f61a16f18820c567c7081606049481ca

Prospective comparison of the Montreal Cognitive Assessment (MoCA) and the Mini Mental State Examination (MMSE) in geriatric oncology

A. Rambeau ${ }^{1}$ B. Beauplet ${ }^{2-3-4}$, H. Laviec ${ }^{3-5,}$, I. Licaj ${ }^{6-7}$, A. Leconte ${ }^{6}$, C. Chatel ${ }^{5-8-9}$, P. Le Bon ${ }^{3-5}$, J. Denhaerynck $^{2}$, B. Clarisse ${ }^{6}$, N. Frenkiel ${ }^{3-8-9}$, M. Lange ${ }^{6-7-10}$ F. Joly ${ }^{1-6-7-10-11}$

${ }^{1}$ Medical oncology department, Centre Francois Baclesse, Caen

${ }^{2}$ Geriatric department, Centre hospitalier universitaire, Caen

${ }^{3}$ Unité de coordination en oncogériatrie de Basse-Normandie, Caen

${ }^{4}$ Normandie Univ, UniCaen, INSERM, U1075, COMETE, 14000 Caen, France

${ }^{5}$ Oncogeriatric department, Centre François Baclesse, Caen

${ }^{6}$ Clinical research department, Centre Francois Baclesse, Caen

${ }^{7}$ Cancer et Cognition Platform, Ligue Nationale contre le Cancer, Caen

${ }^{8}$ Clinical haematology departement, Centre François Baclesse, Caen

${ }^{9}$ Institut d'Hématologie de Basse-Normandie, Caen

${ }^{10}$ Normandie Univ, UniCaen, INSERM, U1086, ANTICIPE, 14000 Caen, France

${ }^{11}$ CHU de Caen, Service d'Oncologie, Caen, France

Corresponding author

Audrey Rambeau

audrey.rambeau@gmail.com

+33695062964

Centre François Baclesse

Avenue du Général Harris

14000 Caen

(C) 2018 published by Elsevier. This manuscript is made available under the CC BY NC user license https://creativecommons.org/licenses/by-nc/4.0/ 


\section{ABSTRACT}

Introduction: Among the various instruments recommended by the International Society of Geriatric Oncology, the Mini Mental State Examination (MMSE) is the most commonly used cognitive screening test before oncological treatment. Although the Montreal Cognitive Assessment (MoCA) has been shown to be more sensitive than the MMSE in several pathologies, no specific data exist for older patients with cancer. We aimed to compare the proportions of older patients with cancer who had screened positive for cognitive impairment according to the MMSE and MoCA scores obtained during a pretherapeutic geriatric assessment (GA) in oncology.

Patients and Methods: This prospective study was conducted among 66 patients older than 70 years who were candidates for a first-line treatment for either a solid tumor or hematological malignancy. Patients with brain tumor or previously known dementia were ineligible. During GA, cognitive function was assessed using both the MoCA and the MMSE tests administered in a random order.

Results: Median age was 78 years. Most patients $(n=43,65.2 \%)$ had a solid tumor. The median scores were 26 [11-30] for MMSE and 24 [8-30] for MoCA. Thirteen (19.7\%) and 44 (66.7\%) patients were screened positive for cognitive impairment according to MMSE and MoCA scores, respectively. Overall, 55 (66.7\%) patients were screened positive: $12(21.8 \%)$ on both scores, $32(70.5 \%)$ with the MoCA only, and one patient with MMSE only $(\mathrm{p}<0.0001)$.

Conclusion: The MoCA test seems to be most relevant to screen for cognitive impairment in older patients with cancer. 


\section{$\underline{\text { INTRODUCTION }}$}

Cognitive impairment is a strong marker of frailty in older persons (1). It is known to be associated with lower survival rates in older patients with cancer $(2,3)$. Cognitive evaluation is therefore an important part of comprehensive geriatric assessment (CGA), as recommended by the International Society of Geriatric Oncology (SIOG). In this context, the SIOG recommends various cognitive screening instruments without specifying any tool to be used in particular (4). Pre-treatment cognitive impairment has been mainly described in older patients with localized breast cancer. According to the literature, the prevalence of cognitive dysfunction ranges between $11 \%$ and $41 \%$ in this population and neuropsychological tests highlight subtle cognitive impairment in most cases (5-9).

Cancer-induced cognitive impairment is an issue that is receiving increasing attention, since cancer and its treatments may impact cognitive performance. Chemotherapy, hormone therapy and antiangiogenic treatments have been shown to be responsible for cognitive disorders (10-12). Mild impairment in episodic memory, working memory, executive functions, attention, and information processing speed are frequently observed. After chemotherapy or hormone therapies, cognitive impairment mostly concerns executive functions (13-15). In addition, older patients exposed to chemotherapy who had lower levels of pre-treatment cognitive reserve also demonstrated a significant decrease in performance on post-treatment processing speed (16). The detection of low cognitive reserve before treatment would influence the risk-benefit ratio of cancer treatment, especially regarding adjuvant chemotherapies.

Additionally, the identification of a pretherapeutic cognitive impairment affects decisions about treatment modalities. As oral chemotherapies are becoming more frequent in oncology, it would be useful to screen pretreatment executive dysfunction. Impaired executive performance is known to major the risk of poor adherence to oral medications (17). Such a relationship was even proven in the case of subtle cognitive impairment among older patients treated by oral cancer agents (18). Furthermore, treatment discontinuation has been associated with higher rates of mortality in older patients with cancer (19). The identification of patients with pre- and posttreatment cognitive impairment using a simple screening tool is therefore a major issue to be able to predict medication adherence and adjust treatment and supportive care in older patients with cancer.

Many tools are available to screen cognitive disorders, such as the Mini-Mental State Examination (MMSE), the clock-drawing test, the Mini-Cog and the Montreal Cognitive Assessment (MoCA) (20). In older patients with cancer who do not have any clinical evidence of dementia, the MMSE and the MoCA are the most widely 
used tools. However, in routine practice, the MMSE remains the most frequently used cognitive impairment screening tool owing to its convenience (21). The MoCA is a ten-minute cognitive screening test validated in more than 50 languages. It explores visuospatial/executive functions, memory, attention, language, abstraction and orientation. It has been shown to be more sensitive than the MMSE in several pathologies such as mild cognitive impairment (22), cerebrovascular involvement (23), Alzheimer's (24) and Parkinson's dementias (25). Although the MoCA includes the assessment of executive functions (alternating trail-making, clock- and cubedrawing), the MMSE does not explore these functions and requires some patients with cancer to perform another test like the clock-drawing test to specifically explore executive functioning. Some studies found that the MoCA was more discriminant than the MMSE in young adults undergoing cancer treatment $(26,27)$ and in patients with malignant brain tumors (28). However, to date, the MMSE and the MoCA tests have not been directly compared in the field of geriatric oncology.

In this context, we compared the proportions of older patients with cancer identified with cognitive impairment using both the MMSE and the MoCA tests performed during a geriatric evaluation prior to any firstline cancer treatment. For the subgroup of patients who received chemotherapy, a second assessment of cognitive performance was performed six to nine months later using both the MMSE and MoCA tests to compare the proportions of cognitive impairment and significant clinical deterioration of cognitive functions compared to baseline.

\section{METHODS}

\section{Study Design}

This prospective bicentric study took place in the Centre François Baclesse cancer hospital and the University Hospital of Caen, from November 2015 to June 2016. The local ethics committee approved the study protocol (Ref. 2014-35, Comité de Protection des Personnes Nord-Ouest III). All patients gave written informed consent. This trial is registered as ID-RCB 2014-A00923-44, clinical trial NCT02558907.

\section{Participants}

The eligibility criteria were as follows: aged 70 years and over; having newly diagnosed localized or metastatic solid cancer or hematological malignancy; candidate for a first-line cancer treatment (chemotherapy, radiation therapy, hormone therapy or surgery); with at least six months life expectancy. Patients with a primary 
or secondary brain tumor, a prevalent dementia or psychiatric pathology that might have impaired cognitive functioning and/or incapacity to answer the cognitive tests (visual, hearing or speaking disabilities, non-French speaker) were not eligible. Patients were offered participation before a CGA or during hospitalization in a chemotherapy ward before initiating cancer treatment.

\section{Assessments and tools}

At baseline, the patients underwent an evaluation of cognitive functions within a complete comprehensive geriatric assessment as recommended by the SIOG, performed by at least a nurse and a geriatrician. Cognitive performance was assessed with the MMSE and the MoCA tests administered in a random order in order to avoid a fatigue effect bias. As both the MMSE and MoCA include similar items for orientation and calculation, they were administered only once in the MMSE. Cognitive impairment was defined as a MMSE score below the $10^{\text {th }}$ percentile, taking into account the educational level (29). Using the MoCA test, cognitive impairment was defined as a total score below $26(22)$.

Routine geriatric characteristics at baseline were assessed as follows: functional status using Activities of Daily Living (ADL) (30), Instrumental Activities of Daily Living (IADL) (31), Timed Up and Go test (32), one-leg standing balance test and history of falling; nutrition using the Mini Nutritional Assessment (MNA) (33); and depression using the 15-item Geriatric Depression Scale (GDS) (34). Polypharmacy and medications that could influence cognitive abilities were collected such as psychotropic, neuroleptic or antidepressant drugs. Comorbidities were rated by the geriatrician using the Cumulative Illness Rating Scale (CIRS-G) (35). When available, the Oncodage G8 frailty screening score was collected (36). Patients were considered at risk of falling if at least one of the following criteria was found: Timed Up and Go test above 20 seconds, one-leg standing balance test less than five seconds, two falls within the last twelve months. Malnutrition was defined as a weight loss of more than $5 \%$ within one month or more than $10 \%$ within six months or a body mass index below 21 $\mathrm{kg} / \mathrm{m}^{2}$, or an albumin blood level below 35g/L or an MNA global score below 17 out of 30. Severe comorbidity was defined as a CIRS-G item score of $\geq 3$. Polypharmacy was defined as five daily medications or more.

For the subgroup of patients who received a first-line chemotherapy, a cognitive assessment was performed by a neuropsychologist six to nine months after the initial CGA at baseline using the MoCA and the 
MMSE tests administered in the opposite order. A significant change in cognitive performance was defined as a modification of $\geq 3$ points from baseline (10\% of the total score) in the MMSE and MoCA scores, respectively.

\section{Statistical analysis}

Assuming that (i) $60 \%$ and $40 \%$ of patients would have cognitive impairment according to the MMSE and the MoCA scores (3), respectively, (ii) $25 \%$ of patients would have cognitive impairment according to the MoCA test but not with the MMSE test and (iii) 5\% of patients with cognitive impairment according to the MMSE test would have cognition disorders with the MoCA test, 60 assessable patients were required (McNemar test, bilateral alpha risk of $5 \%$, power of $80 \%$ ). To anticipate $10 \%$ of non-assessable patients, we decided to include 66 in the present study. Quantitative variables were described with median and range, whereas qualitative variables were described with numbers and percentages. Each patient received the two tests and was matched to himself. We analyzed the matched pairs using the McNemar test for categorical variables with discordant pairs. The Wilcoxon rank-sum test was used for ordinal data and the Chi square or Fisher's exact test for categorical variables. For all tests, a two-tailed $\mathrm{P}$ value lower than or equal to 0.05 was considered statistically significant. We further assessed how patients' characteristics were associated with cognitive impairment detected with at least one of the two tests both at baseline and 6-9 months after treatments. Analyses were performed with STATA, version 12 software (Stata Corp, College Station, TX) and Prism (GraphPad Software, La Jolla, CA).

\section{RESULTS}

\section{Patients characteristics}

The characteristics of the 66 patients included are presented in Table 1. Median age was 78 years [7093]. Patients had been mainly diagnosed with breast cancer $(\mathrm{n}=16,24.2 \%)$, lymphoma $(\mathrm{n}=11,16.7 \%)$ and colorectal cancer $(n=8,12.1 \%)$. Median G8 score was 13 [6-16] $(n=27)$. Patients mostly had a low educational level. Only one patient lived in a nursing home. Vulnerabilities according to the geriatric scale are presented in Table 2. Median ADL and IADL scores were 6 [4-6] and 5 [1-5], respectively. A risk of falling was noted in 56 patients (84.8\%). Malnutrition was found in 26 patients (39.4\%). The median GDS-15 score was 2 [0-15] and 17 patients $(25.8 \%)$ had depressive symptoms according to this questionnaire. 


\section{Baseline cognitive assessment}

Mean MMSE and MoCA scores were 25.7 and 22.3, respectively, corresponding to $19.7 \%$ and $66.7 \%$ of patients with cognitive impairment according to the MMSE and MoCA tests, respectively (McNemar p < 0.0001). Overall, 45 patients $(68.2 \%)$ had cognitive impairment according to at least one cognitive screening test. Among them, twelve patients had impairment on both tests, 32 patients had normal MMSE scores and impairment on the MoCA, and one patient had a normal MoCA score and impairment on the MMSE (Figure 1). According to univariate analysis (Table 3), cognitive impairment on at least one screening test was found to be significantly associated with lower educational levels $(\mathrm{p}<0.01)$ and a trend toward significance with older age $(\mathrm{p}=0.060)$. Psychotropic medication and polypharmacy were also significantly related with poorer cognitive performance ( $\mathrm{p}=0.04$ and $\mathrm{p}<0.001$, respectively). An IADL score under 5 was significantly associated with an increased risk of cognitive impairment $(\mathrm{p}<0.001)$. Compared with patients with a solid tumor, those with a hematological malignancy had better cognitive functions but were also significantly younger (mean age 76.1 and 79.4 years respectively, $\mathrm{p}<0.001)$ and less dependent on IADL $(17.4 \%$ and $46.5 \%$ of dependence respectively $\mathrm{p}=0.031$ ). In addition, no significant difference was found concerning educational level, polypharmacy, and psychotropic treatment between the hematological and solid malignancy subgroups. Of note, the randomized order for taking the tests did not have any impact on cognitive scores $(\mathrm{p}=0.12)$.

\section{Post-chemotherapy cognitive follow-up}

Fiftypatients $(76 \%)$ received chemotherapy, including seventeen for palliative intent. There was no difference in baseline cognitive scores between patients who underwent chemotherapy or not. Among these 50 patients who underwent chemotherapy, nine died during follow-up, ten refused a second cognitive evaluation, three were lost to follow-up and one patient was not assessable. Overall, the post-chemotherapy cognitive follow-up at six to nine months from baseline was performed in 27 patients (seven men and twenty women; median age 74 [70-83] years; eighteen solid cancers (66.7\%) and nine malignant hemopathies (33.3\%)). All the 27 patients received a poly-chemotherapy with a median of 4 [2-12] cycles. According to the various primary cancers, 24 different regimens of chemotherapy were administered, mostly FOLFOX (n=6), docetaxel cyclophosphamide $(n=5)$, FEC100 - paclitaxel $(n=3)$ and R-CHOP $(n=3)$. 
Median MMSE and MoCA scores at follow-up were 28 [22-30] and 25 [19-30], respectively. Overall, 12 patients $(44.4 \%)$ had at least one impaired cognitive test (Figure 2). Among them, three patients had impairment on both tests and nine had normal MMSE scores but impairment on the MoCA. According to the baseline cognitive score, most patients did not suffer from a significant change (at least three points) in cognitive performance after chemotherapy, MMSE and MoCA scores remaining stable for eighteen and twenty-two patients, respectively (Figure 3 ).

Regarding the twelve patients without baseline cognitive impairment whatever the test, the MoCA score showed impairment in one of them, and all MMSE scores remained normal after chemotherapy. Among the fifteen patients with baseline impairment on at least one of the tests, eleven had at least one abnormal score at follow-up (eleven and three with impairment on the MoCA and MMSE scores, respectively). Among patients with at least one abnormal test at follow-up, the majority had no significant change $(n=7)$. No patient had a significant decrease on the MMSE score but two patients had a significant decrease on the MoCA score . Among these two patients, one had no cognitive impairment at baseline. At baseline, the univariate analysis (Table 3) demonstrated a positive relationship between a lower educational level and the risk of cognitive impairment $(\mathrm{p}=0.03)$, as well as a majored risk of cognitive disorders in the event of polypharmacy $(\mathrm{p}=0.02)$.

\section{DISCUSSION}

This study compared the MMSE and MoCA tests to identify cognitive impairment in older patients receiving cancer treatment. Before any oncological treatment, more cognitive impairment was detected with the MoCA than with the MMSE. Furthermore, 32 patients (48\%) had impairment on the MoCA but a normal MMSE score whereas only one patient had impairment on the MMSE but a normal MoCA score. Similar findings were obtained after chemotherapy, with nine patients (33\%) having impairment on the MoCA and a normal MMSE score.

The results of this study are consistent with data from the literature. The mean MoCA score at baseline was 22.3, which is lower than that observed in the normal aging population $(24,37)$ and in young adults with cancer (26-28). However, the mean MoCA score in our population was higher than in subjects with mild cognitive impairment $(\mathrm{MCI})(24,37)$. The same pattern of results was observed for the MMSE score in our population as in the literature. This is consistent with the fact that some of our subjects were likely to have 
undiagnosed mild cognitive impairment. The proportion of patients with an abnormal MMSE score (19.7\%) is similar to that reported in a recent cohort of older patients with cancer (38) and seems representative of oncogeriatric populations. Regarding other geriatric characteristics, the prevalence of malnutrition and depression were similar in our patients and in that cohort. Conversely, our population had fewer severe comorbidities and lower pathological ADL and IADL scores (38). During the enrollment period, the study was proposed to eligible older patients addressed for primary oncologic treatment, but not all specifically addressed for geriatric evaluation, since some patients were recruited during hospitalization in a chemotherapy ward before beginning cancer treatment. These recruitment modalities might explain why some vulnerability factors were less frequent. Although our patients' functional status was good, we found a significant association between cognitive impairment and dependence on the IADL, which are known to be strongly associated (39).

In our population, $66.7 \%$ of patients had cognitive impairment at baseline according to the MoCA as opposed to19.7\% according to the MMSE. In another study, Dubruille et al. (3) found 50\% of pathological MoCA scores and $31 \%$ of pathological MMSE scores in older patients with malignant hemopathy. These high rates emphasize the need to pay systematic attention to cognitive status in aging populations, especially because low cognitive status is known to be associated with poorer survival outcomes $(2,3)$. Indeed, the recent recommendations of the International Society of Geriatric Oncology for prostate cancer management insist on mandatory cognitive screening (40). A recent meta-analysis on cognitive screening tools showed that the MiniCOG and Adenbrooke's Cognitive Evaluation - Revisited tests performed better than the others in screening for dementia, and that the MoCA was better in screening for MCI (20). Since cognitive symptoms are mostly mild in geriatric oncology, using the MoCA test therefore seems particularly relevant.

Unlike the MMSE, the Mini-COG and the MoCA both explore executive functions. As impaired executive functions may lower adherence to oral medication, pretreatment executive dysfunction should be detected before starting an oral therapy (17). Adherence to oral cancer treatment may be reinforced by nursecontrolled administration at home and closer toxicity monitoring (19). Moreover, most of our patients were receiving a psychotropic medication and were polymedicated. Both of these factors have been found to be significantly associated with cognitive impairment. Such medications can decrease sustained attention (41) and may influence executive functioning. This might partly explain why two thirds of our population had an impaired MoCA score. Moreover, polypharmacy is also associated with poorer adherence to oral cancer treatment (42). 
One of the main weaknesses of our study is that cognitive screening results were not compared to a neuropsychological battery of tests, so the MMSE and MoCA performances could not be explored. Moreover, the MMSE assesses educational level whereas the MoCA does not (22,29). In addition, while a third of our patients had a malignant hemopathy, there were no patients with prostate or skin primary cancer who are usually addressed for CGA. In fact, the clinicians were more inclined to propose this study to candidates for a first-line chemotherapy, although the profile of our population did not impact the outcome of the study. Finally, our postchemotherapy follow-up data should be interpreted with caution with regard to the small patients who underwent the second cognitive evaluation.

Post-treatment cognitive impairment was more frequently diagnosed with the MoCA (44.4\%) than the MMSE (11.1\%), so it might be more sensitive than the MMSE for detecting cognitive impairment after chemotherapy. Most patients had no significant cognitive change after chemotherapy whatever the test. Nevertheless, a neuropsychological battery of tests detected cognitive decline in older patients who had received chemotherapy $(9,13-16)$. The fact that we failed to find any significant change in cognitive status after chemotherapy might also be due to the heterogeneity of the regimens followed in our population, so cognitive performances may have been impacted differently (16). Furthermore, it can also be hypothesized that neither the MoCA nor the MMSE are sensitive enough to assess cognitive decline induced by chemotherapy.

In conclusion, cognitive impairment is frequent in geriatric oncology and justifies systematic cognitive screening prior to cancer treatment to optimize the therapeutic decision. Our findings suggest the MoCA might detect more cognitive impairment before and after chemotherapy than the MMSE. Such assumptions warrant a prospective evaluation of the performances of both tests based on the confirmation of cognitive disorders and using a standardized neuropsychological assessment. We have recently begun a prospective study to assess these hypotheses. Its findings may lead to proposals concerning the adaptation of certain practices in geriatric oncology (clinical trial NCT03299855).

\section{ACKNOWLEDGEMENTS}

We wish to thank the patients who participated in this study and the teams in the participating centers. The Northwest Data Center (CTD-CNO) is acknowledged for managing the data. It is supported by grants from the French National League Against Cancer (LNC) and the French National Cancer Institute (INCa). This study 
was supported by a grant from the Cancéropole Nord-Ouest. We are also grateful to Audrey Dugué for her involvement in the study design. 
CONFLICTS OF INTEREST: None to declare

\section{AUTHOR CONTRIBUTIONS}

Conception: Beauplet, Laviec, Clarisse, Leconte, Lange, Joly

Collection and assembly of data: Rambeau, Beauplet, Laviec, Chatel, Lebon, Denhaerynck, Frenkiel, Lange

Data analysis : Licaj

Manuscript writing: Rambeau, Lange, Beauplet, Joly, Licaj

Final approval of manuscript: Rambeau, Beauplet, Laviec, Licaj, Leconte, Chatel, Lebon, Denhaerynck, Clarisse, Frenkiel, Lange, Joly

\section{REFERENCES}

1. Clegg A, Young J, Iliffe S, Rikkert MO, Rockwood K. Frailty in elderly people. Lancet . 2013 Mar 2;381(9868):752-62.

2. Libert Y, Dubruille S, Borghgraef C, Etienne A-M, Merckaert I, Paesmans M, et al. Vulnerabilities in Older Patients when Cancer Treatment is Initiated: Does a Cognitive Impairment Impact the Two-Year Survival? Fardo D, editor. PLoS One. 2016 Aug 1;11(8):e0159734.

3. Dubruille S, Libert Y, Roos M, Vandenbossche S, Collard A, Meuleman N, et al. Identification of clinical parameters predictive of one-year survival using two geriatric tools in clinically fit older patients with hematological malignancies: Major impact of cognition. J Geriatr Oncol. 2015;6(5):362-9.

4. Wildiers H, Heeren P, Puts M, Topinkova E, Janssen-Heijnen MLG, Extermann M, et al. International Society of Geriatric Oncology consensus on geriatric assessment in older patients with cancer. J Clin Oncol. 014 Aug 20;32(24):2595-603.

5. Mandelblatt JS, Stern RA, Luta G, McGuckin M, Clapp JD, Hurria A, et al. Cognitive impairment in older patients with breast cancer before systemic therapy: Is there an interaction between cancer and comorbidity? J Clin Oncol. 2014;32(18):1909-18.

6. Hurria A, Goldfarb S, Rosen C, Holland J, Zuckerman E, Lachs MS, et al. Effect of adjuvant breast cancer chemotherapy on cognitive function from the older patient's perspective. Breast Cancer Res Treat. 2006 Aug 16;98(3):343-8.

7. Lange M, Joly F. How to Identify and Manage Cognitive Dysfunction After Breast Cancer Treatment. $J$ Oncol Pract. 2017 Dec;13(12):784-90.

8. Tchen N, Juffs HG, Downie FP, Yi Q-L, Hu H, Chemerynsky I, et al. Cognitive Function, Fatigue, and Menopausal Symptoms in Women Receiving Adjuvant Chemotherapy for Breast Cancer. J Clin Oncol. 2003;21(22):4175-83. 
9. Lange M, Heutte N, Rigal O, Noal S, Kurtz J-E, Lévy C, et al. Decline in Cognitive Function in Older Adults With Early-Stage Breast Cancer After Adjuvant Treatment. Oncologist. 2016 Nov 29;21(11):1337-48.

10. Joly F, Heutte N, Duclos B, Noal S, Léger-Hardy I, Dauchy S, et al. Prospective Evaluation of the Impact of Antiangiogenic Treatment on Cognitive Functions in Metastatic Renal Cancer. Eur Urol Focus. 2016;2(6):642-9.

11. Joly F, Giffard B, Rigal O, De Ruiter MB, Small BJ, Dubois M, et al. Impact of Cancer and Its Treatments on Cognitive Function: Advances in Research From the Paris International Cognition and Cancer Task Force Symposium and Update Since 2012. J Pain Symptom Manage. 2015 Dec;50(6):83041.

12. Lee PE, Tierney MC, Wu W, Pritchard KI, Rochon PA. Endocrine treatment-associated cognitive impairment in breast cancer survivors: evidence from published studies. Breast Cancer Res Treat. 2016 Aug 18;158(3):407-20. A

13. Lange M, Rigal O, Clarisse B, Giffard B, Sevin E, Barillet M, et al. Cognitive dysfunctions in elderly cancer patients: A new challenge for oncologists. Cancer Treat Rev. 2014 Jul ;40(6):810-7.

14. Gonzalez BD, Jim HSL, Booth-Jones M, Small BJ, Sutton SK, Lin HY, et al. Course and predictors of cognitive function in patients with prostate cancer receiving androgen-deprivation therapy: A controlled comparison. J Clin Oncol. 2015;33(18):2021-7.

15. Hodgson KD, Hutchinson AD, Wilson CJ, Nettelbeck T. A meta-analysis of the effects of chemotherapy on cognition in patients with cancer. Cancer Treat Rev. 2013 May;39(3):297-304.

16. Ahles TA, Root JC, Ryan EL. Cancer- and cancer treatment-associated cognitive change: An update on the state of the science. J Clin Oncol. 2012;30(30):3675-86.

17. Insel K, Morrow D, Brewer B, Figueredo A. Executive Function, Working Memory, and Medication Adherence Among Older Adults. J Gerontol B: Psychol Sci Soc Sci, 61(2):102-107

18. Klepin HD, Geiger AM, Bandos H, Costantino JP, Rapp SR, Sink KM, et al. Cognitive factors associated with adherence to oral antiestrogen therapy: results from the cognition in the study of tamoxifen and raloxifene (Co-STAR) study. Cancer Prev Res 2014 Jan 19;7(1):161-8.

19. Mislang AR, Wildes TM, Kanesvaran R, Baldini C, Holmes HM, Nightingale G, et al. Adherence to oral cancer therapy in older adults: The International Society of Geriatric Oncology (SIOG) taskforce recommendations. Cancer Treat Rev. 2017 Jun;57:58-66.

20. Tsoi KKF, Chan JYC, Hirai HW, Wong SYS, Kwok TCY. Cognitive Tests to Detect Dementia. JAMA Intern Med. 2015 Sep 1;175(9):1450.

21. Folstein MF, Folstein SE, McHugh PR. \&quot;Mini-mental state\&quot;. A practical method for grading the cognitive state of patients for the clinician. J Psychiatr Res . 1975 Nov;12(3):189-98.

22. Nasreddine Z, Phillips N, Bédirian V, Charbonneau S, Whitehead V, Colllin I, et al. The Montreal Cognitive Assessment, MoCA: a brief screening tool for mild cognitive impairment. J Am Geriatr Soc. 2005;53(4):695-9.

23. Pendlebury ST, Cuthbertson FC, Welch SJ V., Mehta Z, Rothwell PM. Underestimation of Cognitive Impairment by Mini-Mental State Examination Versus the Montreal Cognitive Assessment in Patients With Transient Ischemic Attack and Stroke: A Population-Based Study. Stroke. 2010 Jun 1;41(6):1290_ 3 .

24. Freitas S, Simões MR, Alves L, Santana I. Montreal Cognitive Assessment. Alzheimer Dis Assoc Disord. $2013 ; 27(1): 37-43$.

25. Zadikoff C, Fox SH, Tang-Wai DF, Thomsen T, De Bie RMA, Wadia P, et al. A comparison of the mini mental state exam to the Montreal cognitive assessment in identifying cognitive deficits in Parkinson's disease. Mov Disord. 2008;23(2):297-9. 
26. Baxter MF, Dulworth AN, Smith TM. Identification of Mild Cognitive Impairments in Cancer Survivors. Occup Ther Heal Care. 2013 Jan 6;23(7).

27. Arcuri GG, Palladini L, Dumas G, Lemoignan J, Gagnon B. Exploring the measurement properties of the Montreal Cognitive Assessment in a population of people with cancer. Support Care Cancer. 2015;23(9):2779-87.

28. Olson R, Tyldesley S, Carolan H, Parkinson M, Chhanabhai T, McKenzie M. Prospective comparison of the prognostic utility of the Mini Mental State Examination and the Montreal Cognitive Assessment in patients with brain metastases. Support Care Cancer. 2011 Nov 19;19(11):1849-55.

29. Kalafat M, Hugonot-Diener L, Poitrenaud J. Standardisation et étalonnage français du "Mini Mental State"(MMS) version GRECO. Rev Neuropsychol 2003; 13(2), 209-236.

30. Katz S, Ford AB, Moskowitz RW, Jackson BA, Jaffe MW. Studies of Illness in the Aged. JAMA .; 1963 Sep 21;185(12):914.

31. Lawton MP, Brody EM. Instrumental activities of daily living scale (IADL). Gerontologist. 1959;9:17986.

32. Podsiadlo D, Richardson S. The timed Up and Go: a test of basic functional mobility for frail elderly persons. J Am Geriatr Soc . 1991 Feb;39(2):142-8.

33. Guigoz Y, Vellas B, Garry PJ. Assessing the nutritional status of the elderly: The Mini Nutritional Assessment as part of the geriatric evaluation. Nutr Rev. 1996 Jan;54(1 Pt 2):S59-65.

34. Arthur A, Jagger C, Lindesay J, Graham C, Clarke M. Using an annual over-75 health check to screen for depression: validation of the short Geriatric Depression Scale (GDS15) within general practice. Int J Geriatr Psychiatry. 1999 Jun ;14(6):431-9.

35. Linn BS, Linn MW, Gurel L. Cumulative Illness Rating Scale. J Am Geriatr Soc. 1968 May 1;16(5):622-6.

36. Soubeyran P, Bellera C, Goyard J, Heitz D, Curé H, Rousselot H, et al. Screening for vulnerability in older cancer patients: the ONCODAGE Prospective Multicenter Cohort Study. PLoS One .; 2014 Jan;9(12):e115060.

37. Larner AJ. Screening utility of the Montreal Cognitive Assessment (MoCA): in place of - or as well as the MMSE? Int Psychogeriatrics. 2012;24(3):391-6. A

38. Martinez-Tapia C, Canoui-Poitrine F, Bastuji-Garin S, Soubeyran P, Mathoulin-Pelissier S, Tournigand C, et al. Optimizing the G8 Screening Tool for Older Patients With Cancer: Diagnostic Performance and Validation of a Six-Item Version. Oncologist . 2016 Jan 13; 21(2), 188-195.

39. Dodge HH, Kadowaki T, Hayakawa T, Yamakawa M, Sekikawa A, Ueshima H. Cognitive Impairment as a Strong Predictor of Incident Disability in Specific ADL-IADL Tasks Among Community-Dwelling Elders: The Azuchi Study. Gerontologist. 2005 Apr 1;45(2):222-30.

40. Droz J-P, Albrand G, Gillessen S, Hughes S, Mottet N, Oudard S, et al. Management of Prostate Cancer in Elderly Patients: Recommendations of a Task Force of the International Society of Geriatric Oncology. Eur Urol. 2017 Oct 1;72(4):521-31.

41. Stewart SA. The effects of benzodiazepines on cognition. J Clin Psychiatry. 2005;66:9-13.

42. Mathes T, Pieper D, Antoine S-L, Eikermann M. Adherence influencing factors in patients taking oral anticancer agents: A systematic review. Cancer Epidemiol. 2014 Jun 1;38(3):214-26. 
Prospective comparison of the Montreal Cognitive Assessment (MoCA) and the Mini Mental State Examination (MMSE) in geriatric oncology: FIGURES

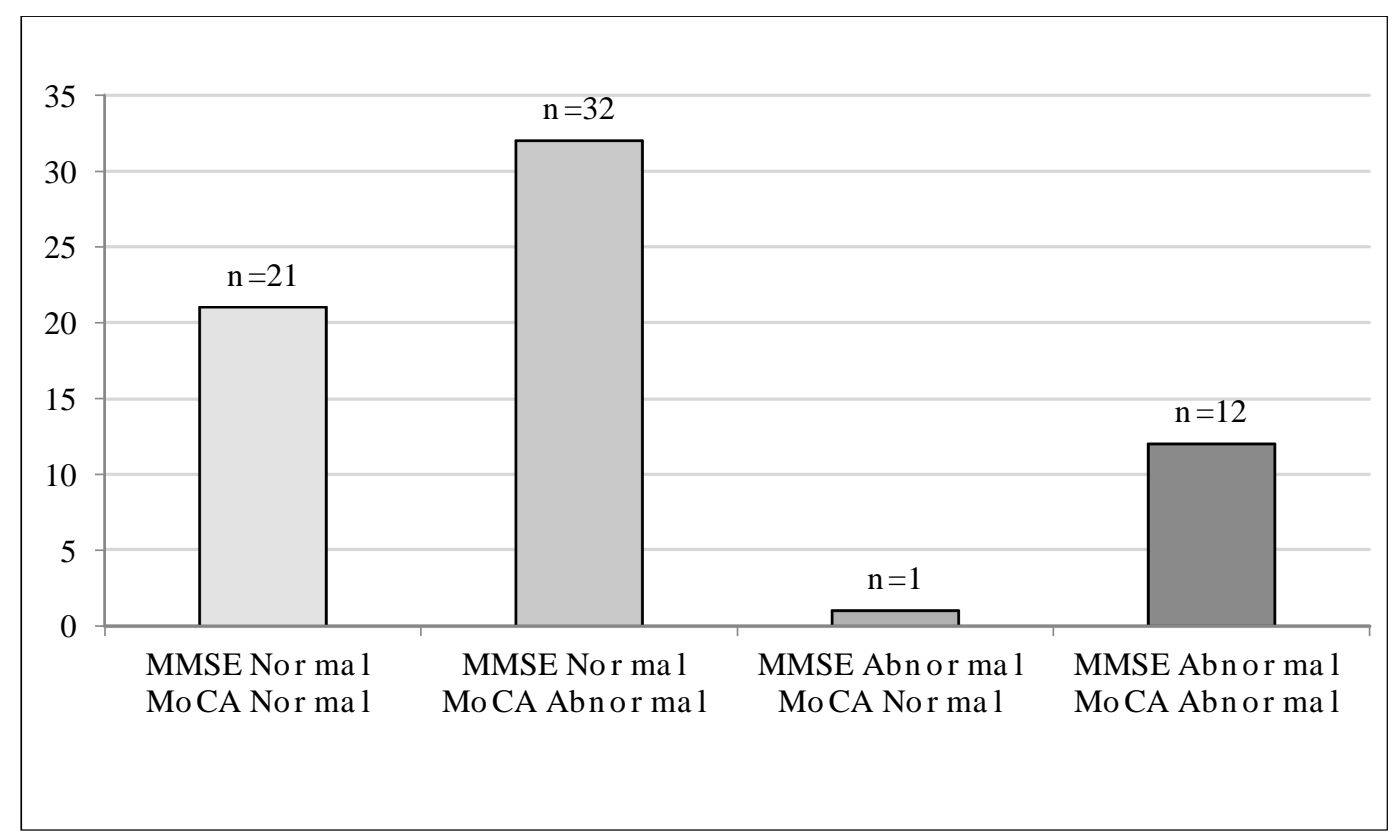

Figure 1. Results of cognitive screening tests at baseline $(n=66)$

Data are presented as number of patients in each subgroup (n)

MMSE: Mini Mental State Examination, MoCA: Montreal Cognitive Assessment 


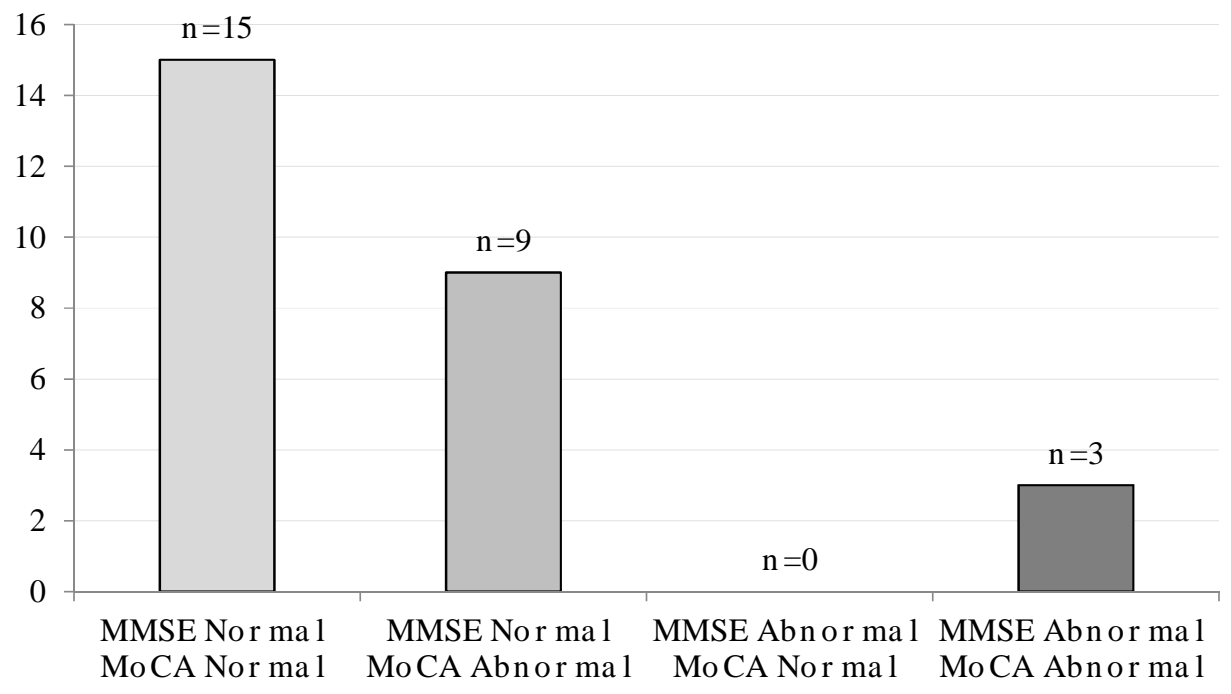

Figure 2. Results of cognitive screening tests at follow-up $(n=27)$

Data are presented as number of patients in each subgroup (n)

Among 66 patients who had baseline assessment, 50 underwent chemotherapy and 27 of them had a cognitive evaluation six to nine months later ( 9 died during follow-up, 10 refused a second cognitive evaluation, 3 were lost to follow-up and one patient was not assessable).

MMSE: Mini Mental State Examination, MoCA: Montreal Cognitive Assessment 


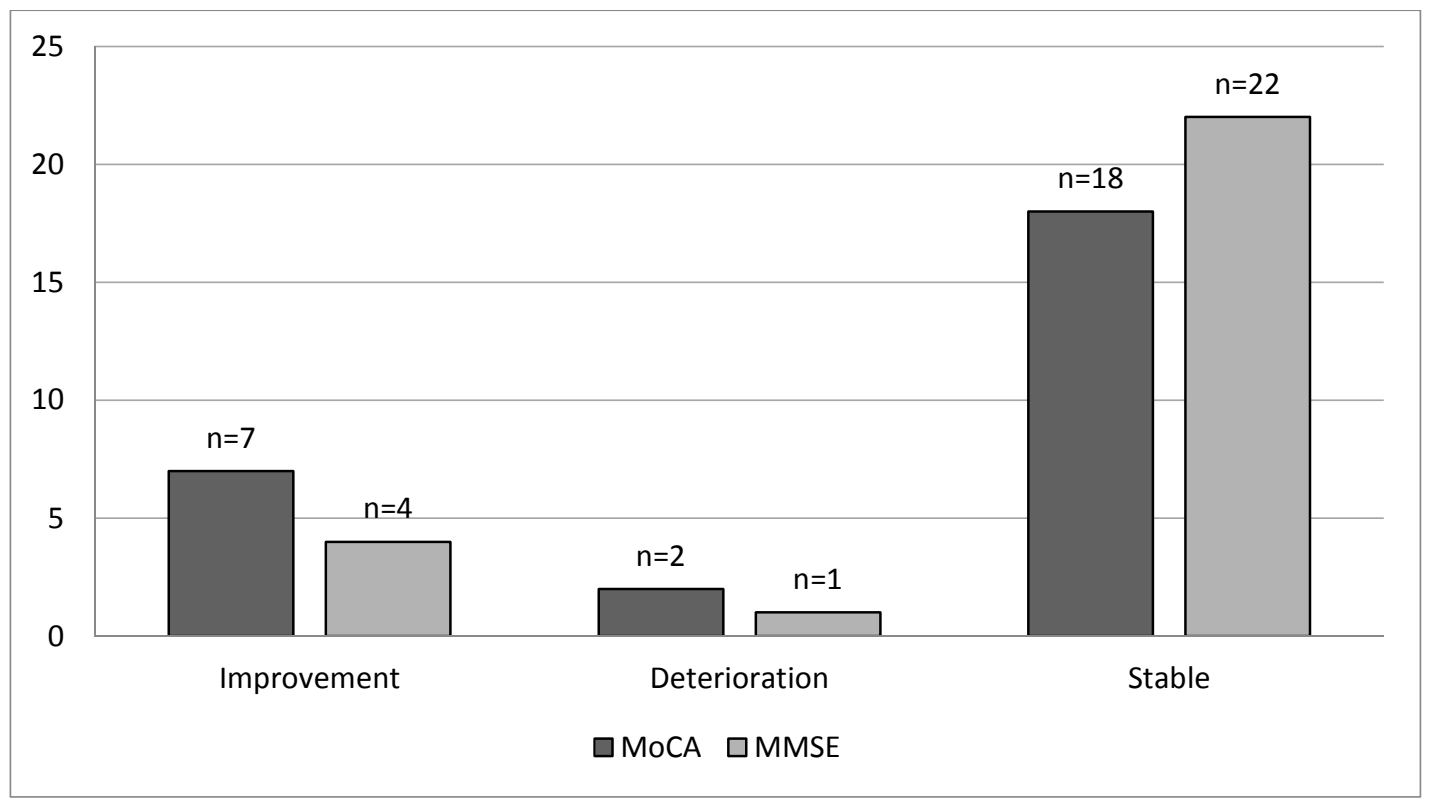

Figure 3. Change between baseline and follow-up for MMSE and MoCA independently (n=27)

Among 66 patients who had baseline assessment, 50 underwent chemotherapy and 27 of them had a cognitive evaluation six to nine months later ( 9 died during follow-up, 10 refused a second cognitive evaluation, 3 were lost to follow-up and one patient was not assessable). Follow up included 27 patients, changes for each test are illustrated above.

An improvement or a deterioration of 3 points or more compared to the initial score was considered significant. If score change was less than 3 points, MMSE and MoCA scores were considered as stable. MMSE: Mini Mental State Examination, MoCA: Montreal Cognitive Assessment 
Prospective comparison of the Montreal Cognitive Assessment (MoCA) and the Mini Mental State Examination (MMSE) in geriatric oncology: TABLES

Table 1. Sociodemographic and medical characteristics at inclusion $(n=66)$

\begin{tabular}{|c|c|c|c|}
\hline & \\
\hline & & $n$ & $\%$ \\
\hline \multirow[t]{2}{*}{ Gender } & Women & 41 & 62.1 \\
\hline & Men & 25 & 37.9 \\
\hline \multicolumn{4}{|c|}{ Age Median 78 [Min 70-Max 93] } \\
\hline & 70-79 years & 41 & 62.1 \\
\hline & 80 years and over & 25 & 37.8 \\
\hline \multirow[t]{4}{*}{$\begin{array}{l}\text { Educational } \\
\text { level }\end{array}$} & Below elementary school & 15 & 22.7 \\
\hline & Elementary school & 30 & 45.5 \\
\hline & Middle school & 15 & 22.7 \\
\hline & High school & 6 & 9.1 \\
\hline \multirow[t]{3}{*}{ Social status } & Alone & 26 & 39.4 \\
\hline & Couple & 39 & 59.1 \\
\hline & Institution & 1 & 1.5 \\
\hline \multicolumn{2}{|c|}{ Risk of falling $^{1}$} & 56 & 84.6 \\
\hline \multicolumn{2}{|c|}{ Severe comorbidity $^{2}$} & 5 & 7.6 \\
\hline \multirow{3}{*}{$\begin{array}{l}\text { Number of } \\
\text { medications }\end{array}$} & $0-4$ & 24 & 36.4 \\
\hline & $5-9$ & 34 & 51.5 \\
\hline & 10 and more & 8 & 12.1 \\
\hline \multirow{5}{*}{$\begin{array}{l}\text { Psychotropic } \\
\text { medication }\end{array}$} & Total & 39 & 59.1 \\
\hline & Benzodiazepine & 18 & 27.3 \\
\hline & Neuroleptic & 2 & 3.0 \\
\hline & Antidepressant & 5 & 7.6 \\
\hline & Morphinic or codeine & 14 & 21.2 \\
\hline \multicolumn{2}{|l|}{ Malnutrition } & 26 & 39.4 \\
\hline \multirow[t]{9}{*}{ Cancer } & Solid tumor, including : & 43 & 65.2 \\
\hline & Breast & 16 & 24.2 \\
\hline & Colorectal & 8 & 12.1 \\
\hline & Gynecologic & 4 & 6,1 \\
\hline & Skin & 3 & 4.5 \\
\hline & Hemopathy, including : & 23 & 34.8 \\
\hline & Lymphoma & 11 & 16.7 \\
\hline & Acute leukemia & 7 & 10.6 \\
\hline & Myeloma & 3 & 4.5 \\
\hline
\end{tabular}

${ }^{1}$ Were considered at risk for falling if at least one of the following criteria was found: Timed Up and Go test less than 20 seconds, one-leg standing balance test less than 5 seconds or 2 falls during the last 12 months

${ }^{2}$ Severe comorbidity was defined as a CIRS-G item score of $\geq 3 / 4$ 
Table 2. Vulnerability of patients according to geriatric scale in comprehensive geriatric assessment at baseline

\begin{tabular}{|c|c|c|c|c|}
\hline & Cut-off score & Median & Range & Vulnerable $\%$ \\
\hline G8 Oncodage (39) & $\leq 14 / 17$ & 13 & $6-16$ & $37 \%$ \\
\hline \multicolumn{5}{|l|}{ Functional status } \\
\hline ADL (33) & $<6 / 6$ & 6 & $4-6$ & $22.7 \%$ \\
\hline IADL (34) & $<5 / 5$ & 5 & $1-5$ & $36.4 \%$ \\
\hline \multicolumn{5}{|l|}{ Emotional status } \\
\hline GDS 15 (37) & $\geq 5 / 15$ & 2 & $0-15$ & $25.8 \%$ \\
\hline \multicolumn{5}{|l|}{ Cognitive status } \\
\hline MMSE (21) & $<$ Percentile $10^{*}$ & 26 & $11-30$ & $19.7 \%$ \\
\hline MoCA (22) & $\leq 26 / 30$ & 24 & $9-30$ & $66.7 \%$ \\
\hline
\end{tabular}

G8 oncodage is a geriatric frailty screening score in oncology, which cut-off $\leq 14 / 17$ predicts abnormal CGA

ADL: 6-items Activities of Daily Living, a score less than 6 indicates autonomy difficulties

IADL: 5-items Instrumental Activities of Daily Living, a score less than 5 indicates instrumental autonomy difficulties

GDS: Geriatric Depression Scale 15 items, a score that 5 or more indicated depressive symptoms depression.

MMSE: Mini Mental Statement Examination, MMSE cut-off was different according to educational level. *Percentile 10 cut-off score was $24 / 30$ for level 1 (below elementary school or elementary school without diploma), 25/10 for level 2 (elementary school with diploma or middle school without the last year), 26/30 for level 3 (last year of middle school or high school without diploma) and 27/30 for level 4 (high school with diploma and more). In patients older than 80, cut-off score was 1 point lower than previously described cut-off (29).

MoCA: Montreal Cognitive Assessment 
Table 3. Characteristics at baseline and follow-up associated with positive screening for cognitive impairment with at least one cognitive test

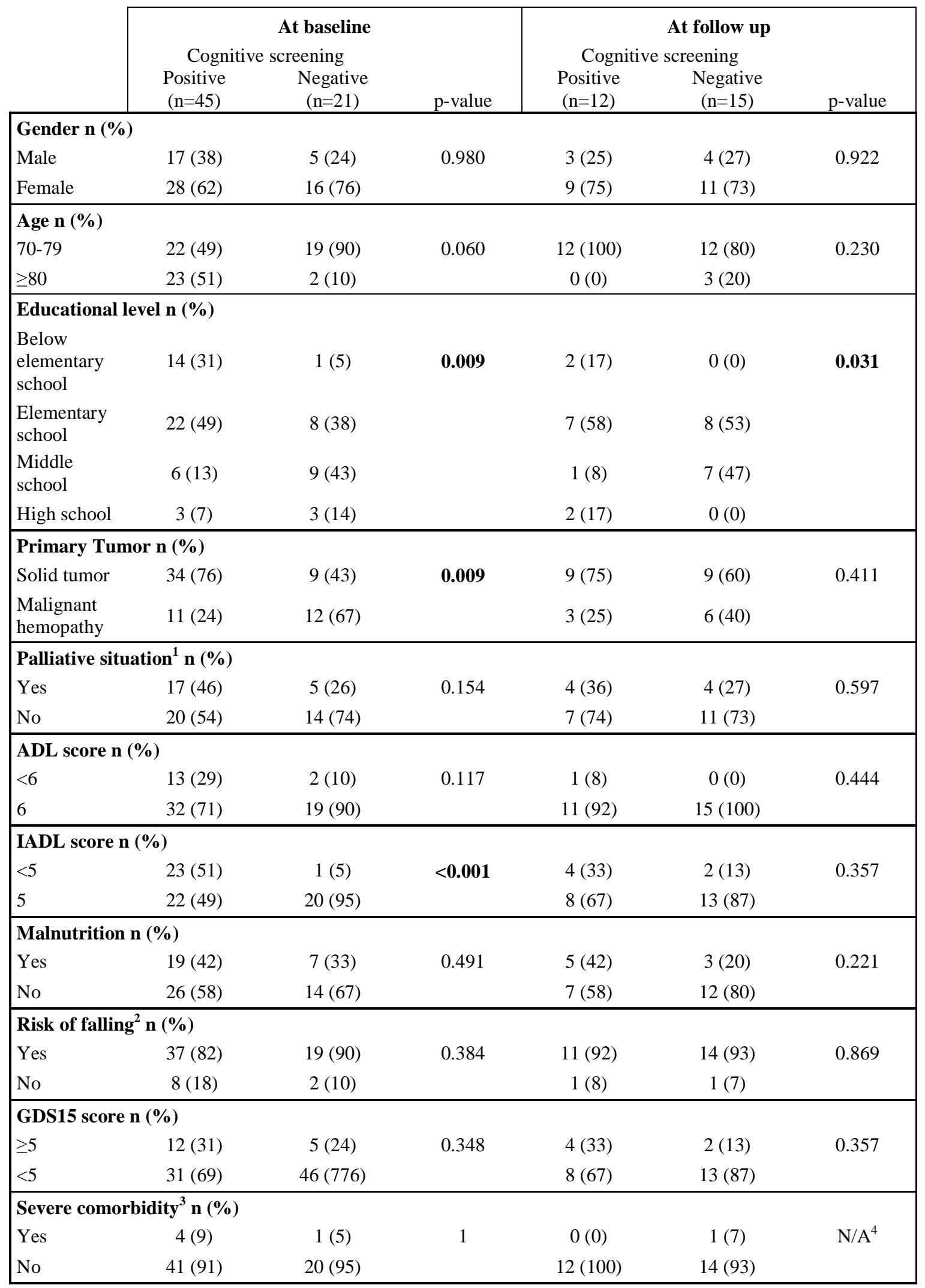




\begin{tabular}{|c|c|c|c|c|c|c|}
\hline \multicolumn{7}{|c|}{ Number of medications $n(\%)$} \\
\hline$\geq 5$ & $36(80)$ & $6(29)$ & $<0.001$ & $9(75)$ & $4(27)$ & 0.021 \\
\hline$<5$ & $9(20)$ & $15(71)$ & & $3(25)$ & $11(73)$ & \\
\hline \multicolumn{7}{|c|}{ Psychotropic medication $^{5}$ n (\%) } \\
\hline Yes & $27(60)$ & $7(33)$ & 0.043 & $7(58)$ & $4(27)$ & 0.096 \\
\hline No & $18(40)$ & $14(67)$ & & $5(42)$ & $11(73)$ & \\
\hline \multicolumn{7}{|c|}{ Test assessment order $\mathrm{n}(\%)$} \\
\hline MMSE first & $25(56)$ & $8(38)$ & 0.290 & $8(67)$ & $9(60)$ & 0.252 \\
\hline MoCA first & $20(44)$ & $13(62)$ & & $4(33)$ & $6(40)$ & \\
\hline
\end{tabular}

Chi square and Fisher's exact tests were used comparing proportions of patient screened positive or not for cognitive impairment. A two tailed $\mathrm{p}$ value $>0.05$ was considered as significant. Screening was positive in $n=45 / 66$ at baseline and $n=12 / 27$ at follow-up.

ADL: 6-items Activities of Daily Living, IADL: 5-items Instrumental Activities of Daily Living, GDS15: Geriatric Depression Scale 15 items, MMSE: Mini Mental State Examination, MoCA: Montreal Cognitive Assessment

${ }^{1}$ Palliative situation: Non-curative intent of treatment

${ }^{2}$ Were considered at risk for falling if at least one of the following criteria was found: Timed Up and Go test above 20 seconds, one-leg standing balance test less than 5 seconds, 2 falls during the last 12 months

${ }^{3}$ Severe comorbidity was defined as a CIRS-G item score of $\geq 3 / 4$

${ }^{4}$ Value non-calculable

${ }^{5}$ Psychotropic medication includes benzodiazepins, antidepressants, opioids 